\title{
Optimal Probabilistic Scheduling in Time Slotted Multiple Access
}

\author{
KONSTANTINOS DEMESTICHAS, EVGENIA ADAMOPOULOU \\ Institute of Communication and Computer Systems \\ National Technical University of Athens \\ Iroon Polytechneiou 9, Zografou 15773 \\ GREECE
}

\begin{abstract}
This paper presents an efficient scheduling model for the delivery of sensing data in networks that use time division multiple access. The model is capable of achieving the optimal solution in terms of total delivery time, given certain constraints on radio resources. The proposed solution adopts a probabilistic approach which is based on a problem formulation utilizing chained binomial distributions.
\end{abstract}

Key-Words: binomial distribution, multiple access, probabilistic model, radio resources, sensing reports, scheduling, time slots.

Received: June 9, 2020. Revised: December 1, 2020. Accepted: December 14, 2020. Published: December 24, 2020.

\section{Introduction}

In our days, community-based mobile applications have become quite popular and have enjoyed considerable market penetration. This trend is initially seen as a natural evolution of existing Web-based counterparts, e.g. mobile social networks. Beyond this, however, a community of mobile users may be perceived not only as a mobile social network, but also as a highly distributed sensor network that may be exploited to properly monitor ("sniff") its radiofrequency (RF) or physical environment. This concept has been initiated and studied already from the early days of packet-based mobile networks [1]-[2], as well as more recently in the context of mobile phone sensors networks [3], the Internet of Things (IoT) [4], and mobile crowd sensing [5]. For instance, such types of sensing data can be of particular importance for cognitive networks and applications, which need to continuously monitor their environment so as to make proper adaptations [6]. We may refer to the first group of mobile community services as "social network applications", whereas to the second one as "sensor-like applications", which constitute the focal point of this paper.

The main notion behind sensor-like applications is the ability to issue a query for -usually- impersonalized information that resides in a community of mobile or sensing terminals. This information may vary both in size and nature. It may range from a few hundred bytes to a few Megabytes. It may include various terminal-monitored parameters, such as: the perceived signal strength level of the base station or access point the terminal is attached to, or of neighbouring stations; the interference temperature levels within frequency bands of interest; the terminal velocity (retrievable through Global Positioning System - GPS) or acceleration; the temperature or humidity levels in the terminal's area; various layer-2, layer-3 and layer-4 historical measurements of the terminal operation; miscellaneous multimedia feedback, such as images, audio, videos, or text; and more. Applications can be numerous and widely heterogeneous. For instance, the introduced technique can be applied in wireless sensors networks in the IoT for precision agriculture [7]-[8].

These sensor-like mobile community applications involve the simultaneous response of multiple (e.g. ranging from just a handful of terminals up to several hundreds) mobile terminals belonging to a specific "sensing" community [9]. Thus, a major attribute of the aforementioned reporting process is the simultaneous utilization of uplink channels, by multiple sensing terminals, which have the same or similar data payload to transmit. A generalization for cases where data payloads are unequal can also be 
performed. Such procedure is regularly triggered, i.e. with a potentially high frequency of execution. Notably it, in a cognitive radio context [10], it might consume part of the same radio resources that are used for serving regular user traffic (voice, data, etc.). Hence, the reporting process should achieve two goals:

- The collection of all sensing data must be performed as fast as possible, or, in other words, with the minimum possible total delay (since a significant delay would render the data outdated).

- The completion of the process must be carried out by respecting specific resource constraints imposed by the network (so as to leave as many resources available for other uses, including the regular user traffic).

A simple approach would be to enable the sensing terminals to randomize their transmissions, with a view to avoiding an excessive impact on the radio resources. This paper formulates and highlights the policies that must be followed by such a randomization process, so as to reach the optimal solution.

Hence, in this paper, we present a scheduling algorithm time slotted networks, such as for generalized Time Division Multiple Access (TDMA) based networks [12], where the imposed resource constraints correspond to the number of channels that can be used by the sensing terminals. In particular, the network resource management might impose that only $k$ (e.g., $k=1,2, \ldots)$ sensing terminals are permitted to simultaneously transmit at a time. Thus, only $k$ "resources" are available for the sensing reporting process at each point in time, i.e. only $k$ sensing transmissions per time interval are allowed (for instance, this can be achieved by means of combining spatial reuse TDMA or another technique [11]). The algorithm proposed herein is based on a chained binomial distribution scheduling $(C B D S)$ model. The paper first demonstrates the proposed solution for the simple case of $k=1$ (for the purpose of easier comprehension), and then produces a generalization (i.e. for $k \geq 1$ ).

The proposed scheduling algorithm presents the following desired characteristics:

- It consists of an indirect, rather than an explicit, scheduling technique, meaning that the server or gateway where the sensing data are gathered does not need to instruct the sensing terminals one by one, regarding the initiation and the completion of their transmissions. Such an instruction process would lead to an overload of the downlink signaling channels. In contrast, by enabling the sensing terminals to configure their transmission times by themselves, the network conserves resources, and there is no need for implementation a complex scheduler.

- The introduction of new signaling channels is avoided and the use of the application layer is possible.

\section{Background and Problem Solution}

\subsection{Background}

The binomial distribution represents the discrete probability distribution of the occurrence of exactly $i$ successful results out of $n$ Bernoulli experiments [13]. In Bernoulli experiments, the probability of a separate experiment being "successful" is $q$, whereas the probability of being "unsuccessful" is $p=1-q$. Based on this notation, the probability $Q(i, n)$ of $i$ successful results out of $n$ experiments is:

$$
Q(i, n)=\left(\begin{array}{l}
n \\
i
\end{array}\right)(1-q)^{n-i} q^{i}
$$

Let us now assume a TDMA system with $n$ operating sensing terminals. Assuming that every terminal has one packet of sensing report data to transmit, then, according to (1), the probability of $i$ terminals out of $n$ transmitting within a TDMA timeslot is:

$$
Q_{r}(i, n)=\left(\begin{array}{l}
n \\
i
\end{array}\right)\left(1-q_{r}\right)^{n-i} q_{r}^{i}
$$

where $Q_{r}(i, n)$ is the aforementioned probability, and $q_{r}$ the probability of packet transmission by a terminal. Without loss of generality, it is assumed that each packet requires a single timeslot to be transmitted, and that every sensing terminal has a single packet to transmit, although the same model can also be used in cases of multiple required timeslots or multiple packets.

A successful transmission is followed by the withdrawal of the corresponding terminal from the current transmission process, until at least a new sensing report cycle is initiated. Each terminal selects in a random way whether to transmit the composed sensing packet in a certain timeslot or not. We seek the optimal policy $q_{r}=q_{r}(n)$ that a terminal must follow, in order for the collection of all reports to be completed in the minimum time possible. The required solution must be symmetrical and fair, by not distinguishing the terminals into primary, secondary, etc., classes. In other words, the same transmission 
policy $q_{r}=q_{r}(n)$ must be followed by all terminals.

\subsection{Problem Formulation and Solution}

Initially, we assume that only one (or none) transmission per timeslot is allowed. This means that if two or more simultaneous transmissions coincide, then none of them is successful and the timeslot is rendered unutilized. The abovementioned problem can be modeled as depicted in Fig. 1.

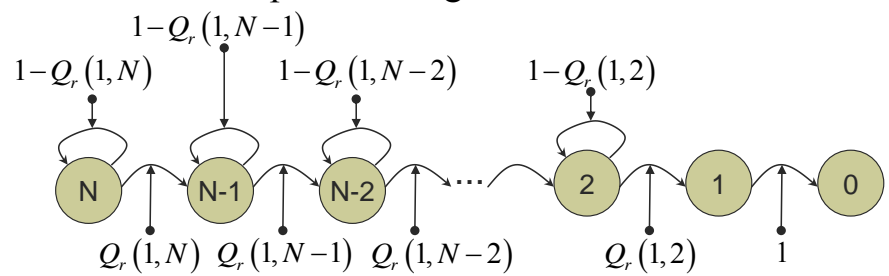

Fig. 1. CBDS model for one permitted transmission per timeslot

As becomes obvious from Fig. 1, there are two possibilities in every state: either to remain in the current state with a probability of $1-Q_{r}(1, n)$, where $n$ denotes the current state (i.e., the number of sensing terminals that have not yet successfully completed their transmission), or to transit in state $n-1$ with a probability of $Q_{r}(1, n)$. We seek the optimal policy $q_{r}=q_{r}(n)$ which minimizes the first probability and maximizes the second one. It is obvious that the solution which minimizes the first probability coincides with the solution that maximizes the second one, since:

$$
\begin{gathered}
\frac{d}{d q_{r}}\left\{1-Q_{r}\left(1, n, q_{r}(n)\right)\right\}=-\frac{d Q_{r}(1, n)}{d q_{r}} \\
\frac{d}{d q_{r}}\left\{Q_{r}\left(1, n, q_{r}(n)\right)\right\}=\frac{d Q_{r}(1, n)}{d q_{r}}
\end{gathered}
$$

Consequently, the problem can now be expressed as follows: We seek the function $q_{r}=q_{r}(n)$ which satisfies the following condition:

$$
\begin{aligned}
& \frac{d Q_{r}(1, n)}{d q_{r}}=0, \forall n>1, \\
& \text { with } q_{r}=q_{r}(n) \in(0,1)
\end{aligned}
$$

It holds that:

$$
\begin{gathered}
Q_{r}(1, n)=n\left(1-q_{r}\right)^{n-1} q_{r} \\
\frac{d Q_{r}(1, n)}{d q_{r}}=n\left(1-q_{r}\right)^{n-2}(1 \\
\left.-q_{r} n\right)
\end{gathered}
$$

From (7), due to (5), it is deduced that:

$$
q_{r}(n)=\frac{1}{n}
$$

Equation (8) represents the optimal transmission policy when only one transmission per timeslot is permitted, and constitutes the mathematical proof of the intuitive solution that is often applied among $n$ equal competitors in order to minimize their conflicts. According to this equation, the probability of the transmission policy should be inversely proportional to the number of sensing terminals with pending transmissions. This specific solution gives insight to the more general and complex one, that follows.

We will now present a generalization of the proposed model, in case $k>1$ simultaneous transmissions are permitted. In this case, the model depicted in Fig. 2 is applied, which is an extension of the model presented in Fig. 1.

As may be observed from Fig. 2, the possibilities now in each state are $k+1$, where $k$ is the maximum number of permitted simultaneous transmissions. The first possibility is to stay in the current state $n$. The remaining possibilities correspond to the occurrence of $1,2, \ldots, k$ simultaneous transmissions.

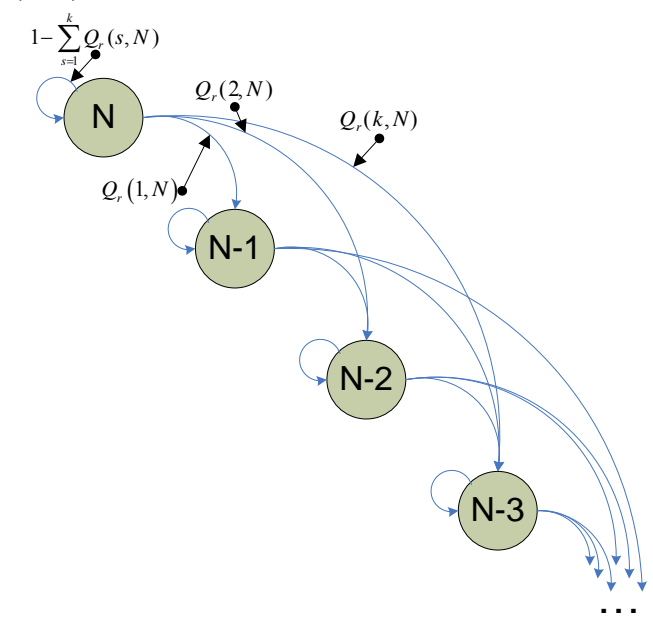

Fig. 2. CBDS model for $\mathrm{k}>=1$ (graph corresponds to $\mathrm{k}=3$ )

The probability of staying in the current state $n$ is now expressed by:

$$
Q_{p}(k, n)=1-\sum_{s=1}^{k} Q_{r}(s, n)
$$

The goal is to minimize the abovementioned probability, thus the following condition must be satisfied:

$$
\begin{gathered}
\frac{d}{d q_{r}}\left\{1-\sum_{s=1}^{k} Q_{r}(s, n)\right\}=0, \forall n>1, \\
\text { with } q_{r}=q_{r}(n) \in(0,1)
\end{gathered}
$$




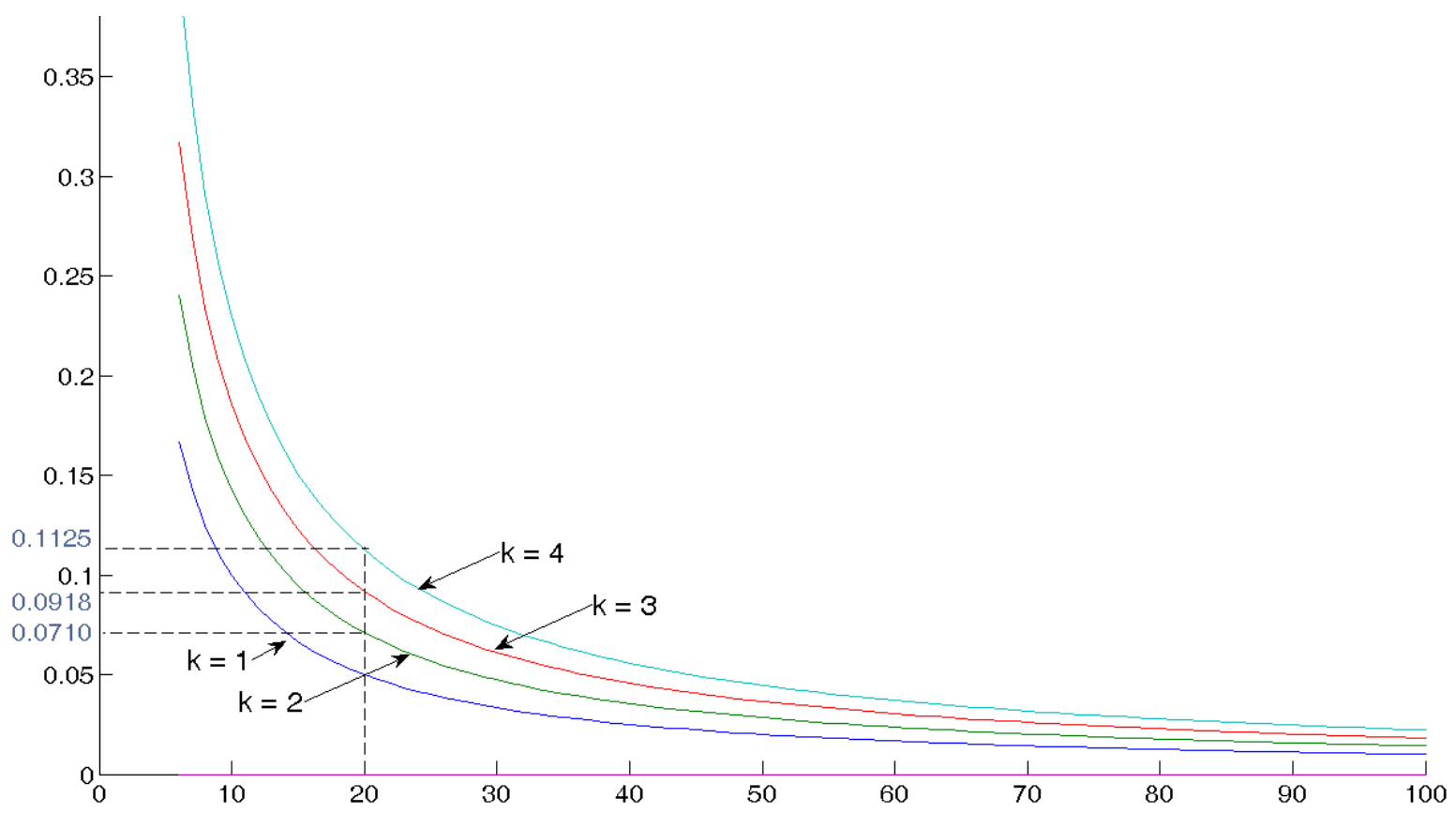

We seek, given the value of parameter $k$, the policy $q_{r}=q_{r}(n)$ that satisfies equation (10). Based on (10), it follows that:

$$
\sum_{s=1}^{k} \frac{d}{d q_{r}}\left\{Q_{r}(s, n)\right\}=0
$$

It also holds that:

$$
\begin{gathered}
Q_{r}(s, n)=\left(\begin{array}{l}
n \\
s
\end{array}\right)\left(1-q_{r}\right)^{n-s} q_{r}{ }^{s} \\
\frac{d Q_{r}(s, n)}{d q_{r}}=\left(\begin{array}{l}
n \\
s
\end{array}\right)\left(1-q_{r}\right)^{n-s-1} q_{r}^{s-1}\left(s-n q_{r}\right)
\end{gathered}
$$

Fig. 3. Results for different values of permitted simultaneous transmissions per timeslot and different numbers of terminals

Hence, from (11) and (13), it is deduced that:

$$
\sum_{s=1}^{k}\left(\begin{array}{l}
n \\
s
\end{array}\right)\left(1-q_{r}\right)^{n-s-1} q_{r}^{s-1}\left(s-n q_{r}\right)=0
$$

or equivalently:

$$
\sum_{s=1}^{k}\left(\begin{array}{l}
n \\
s
\end{array}\right)\left(1-q_{r}\right)^{k-s} q_{r}^{s-1}\left(s-n q_{r}\right)=0
$$

The optimal transmission policy $q_{r}=q_{r}(n)$, given the maximum number of permitted transmissions $k$ and the number of pending sensing terminals $n$, can be determined by solving equation (15).

In practice, the reporting terminals can retrieve the value of parameter $k$ and the current value of $n$, through the downlink broadcast channel. The terminals do not have to solve equation (15) in realtime but can instead retrieve its solution from a $k \times n$ look-up table. Equivalently, this solution can be announced by the server via the broadcast channel.

In the following section, the solutions for the problem of the determination of the optimal transmission policy, for different values of permitted transmissions per timeslot, $k$, are presented. These solutions have been determined by solving equation (15) numerically, and are depicted in Fig. 3. The horizontal axis represents the number of sensing terminals with pending transmissions, while the vertical axis represents the optimal probability of retransmission.

\section{Numerical results}

For instance, when there are $n=20$ sensing terminals with pending transmissions, the optimal transmission policy for each of the terminals is to transmit with a probability of $q_{r}=0.05$ if $k=1, q_{r}=0.071$ if $k=2$, $q_{r}=0.0918$ if $k=3, q_{r}=0.1125$ if $k=4$, etc. Any other choice for the value of $q_{r}$ is sub-optimal. As expected, as the number of sensing terminals decreases, the probability of the optimal transmission policy increases. Furthermore, when more simultaneous transmissions are permitted, the optimal transmission probabilities are increased, for the same number of sensing terminals.

\section{Conclusion}

In this paper, we presented an efficient probabilistic scheduling model for the reporting of sensing data, in TDMA based networks. The goal of the model was to 
minimize the time required to gather all sensing reports, from multiple sensing terminals, while conforming to specific resource constraints. The scheduling problem was mathematically formulated and solved, based on the proposed probabilistic approach named CBDS. Applications of this technique are wide-ranging, especially wherever there needs of scheduling (e.g., refer to [14]-[16]) a highly concentrated number of sensing terminals in a streamlined and distributed manner.

\section{References:}

[1] E. Adamopoulou, K. Demestichas, C. Dessiniotis, J. Markoulidakis, and M. Theologou, "Introducing Anonymous Real-Time Mobile Community Services," in 2007 16th IST Mobile and Wireless Communications Summit, Jul. 2007, pp. 1-5, doi: 10.1109/ISTMWC.2007.4299122.

[2] K. P. Demestichas, E. F. Adamopoulou, J. G. Markoulidakis, and M. E. Theologou, "Towards Anonymous Mobile Community services," Journal of Network and Computer Applications, vol. 32, no. 1, pp. 116-134, Jan. 2009, doi: 10.1016/j.jnca.2008.04.002.

[3] V.-D. Le, "Distributed opportunistic sensing in mobile phone sensor networks," in 2013 IEEE International Conference on Pervasive Computing and Communications Workshops (PERCOM Workshops), Mar. 2013, pp. 427-428, doi: 10.1109/PerComW.2013.6529535.

[4] J. Jin, J. Gubbi, T. Luo, and M. Palaniswami, "Network architecture and QoS issues in the internet of things for a smart city," in 2012 International Symposium on Communications and Information Technologies (ISCIT), Oct. 2012, pp. 956-961, doi: 10.1109/ISCIT.2012.6381043.

[5] Z. Xu et al., "Mobile crowd sensing of human-like intelligence using social sensors: A survey," Neurocomputing, vol. 279, pp. 3-10, Mar. 2018, doi: 10.1016/j.neucom.2017.01.127.

[6] S. Haykin, "Cognitive Radio: Brain-Empowered Wireless Communications", IEEE JSAC, vol. 23, no. 2, pp. 201-220, Feb. 2005.

[7] E.-S. E. Omran and A. M. Negm, "Smart Sensing System for Precision Agriculture," in Technological and Modern Irrigation Environment in Egypt: Best Management Practices \& Evaluation, E.-S. E. Omran and A. M. Negm, Eds. Cham: Springer International Publishing, 2020, pp. 77-105.
[8] N. Ahmad, A. Hussain, I. Ullah, and B. H. Zaidi, "IOT based Wireless Sensor Network for Precision Agriculture," in 2019 7th International Electrical Engineering Congress (iEECON), Mar. 2019, pp. 1-4, doi: 10.1109/iEECON45304.2019.8938854.

[9] K. Demestichas, E. Adamopoulou, and $M$. Theologou, "Application-Layer CommunityOriented Uplink Scheduler for CDMA Networks", IJCS, vol. 22, no. 5, pp. 641-649, May 2009, https://doi.org/10.1002/dac.993.

[10]E. Adamopoulou, K. Demestichas, and M. Theologou, "Enhanced estimation of configuration capabilities in cognitive radio," IEEE Communications Magazine, vol. 46, no. 4, pp. 56-63, Apr. 2008, doi: 10.1109/MCOM.2008.4481341.

[11] J. Gronkvist, "Assignment methods for spatial reuse TDMA," in 2000 First Annual Workshop on Mobile and Ad Hoc Networking and Computing. MobiHOC (Cat. No.00EX444), Aug. 2000, pp. 119-124, doi: 10.1109/MOBHOC.2000.869219.

[12] J. Ma, W. Lou, Y. Wu, X.-Y. Li, and G. Chen, "Energy Efficient TDMA Sleep Scheduling in Wireless Sensor Networks," in IEEE INFOCOM 2009, Apr. 2009, pp. 630-638, doi: 10.1109/INFCOM.2009.5061970.

[13] A. Papoulis, Probability, Random Variables, and Stochastic Processes, 2nd ed. New York: McGraw-Hill, pp. 102-103, 1984.

[14] Yingwei Yao and G. B. Giannakis, "Energyefficient scheduling for wireless sensor networks," IEEE Transactions on Communications, vol. 53, no. 8, pp. 1333-1342, Aug. 2005, doi: 10.1109/TCOMM.2005.852834.

[15] Text B. Yu, J. Li, and Y. Li, "Distributed Data Aggregation Scheduling in Wireless Sensor Networks," in IEEE INFOCOM 2009, Apr. 2009, pp. 2159-2167, doi: 10.1109/INFCOM.2009.5062140.

[16] M. Chitnis, P. Pagano, G. Lipari, and Y. Liang, "A Survey on Bandwidth Resource Allocation and Scheduling in Wireless Sensor Networks," in 2009 International Conference on Network-Based Information Systems, Aug. 2009, pp. 121-128, doi: 10.1109/NBiS.2009.77. 


\section{Contribution of individual authors to the creation of a scientific article (ghostwriting policy)}

Konstantinos Demestichas initiated the concept and prepared the mathematical problem formulation and its solution in Section 2.

Evgenia Adamopoulou updated the mathematical formulation, run the numerical results in Section 3, and carried out the state-of-the-art survey and the motivation description in Section 1.
Creative Commons Attribution License 4.0 (Attribution 4.0 International, CC BY 4.0)

This article is published under the terms of the Creative Commons Attribution License 4.0

https://creativecommons.org/licenses/by/4.0/deed.en US 